\title{
Design, performance, and limitations of fibers for cladding-pumped Raman lasers
}

\author{
Johan Nilsson ${ }^{\text {a, b }}$, Junhua Ji ${ }^{\text {a }}$, Christophe A. Codemard ${ }^{\text {a, }}$, and Jayanta K. Sahu ${ }^{a}$ \\ ${ }^{a}$ Optoelectronics Research Centre, University of Southampton, SO17 1BJ, UK \\ b jn@orc.soton.ac.uk \\ ${ }^{\mathrm{c}}$ Present address Fianium Ltd., Southampton, SO31 4RA, UK
}

\begin{abstract}
We discuss designs of double-clad Raman fiber lasers for efficient $1^{\text {st }}$-Stokes generation. Limiting factors include unwanted $2^{\text {nd }}$ Stokes generation, material damage, dispersion, inner-cladding NA, and background loss. A brightness enhancement of over 2000 is possible.
\end{abstract}

Keywords: Optical fiber amplifiers, Raman scattering, Raman lasers.

Cladding-pumped (CP) fiber Raman lasers and amplifiers represent a new concept to obtain Raman gain and power conversion in a single-mode core, whilst still allowing for multimode pumping [1,2]. The result combines many attractions of the hugely successful cladding-pumped rare-earth doped fiber with the versatility and flexibility of Raman gain. Thus, a multimode pump beam launched into a "passive" double-clad Raman fiber (DCRF) with core and inner cladding made of silica glass can be converted into a (nearly) diffraction-limited output signal beam propagating in the core through stimulated Raman scattering (SRS). Therefore, CP fiber Raman devices enhance the brightness, and the scope for brightness enhancement (BE) is a primary consideration just as for CP rare-earth (RE) doped fibers and many other optically pumped lasers.

While SRS provides wavelength flexibility and many other benefits relative to RE-doped fibers, there are also many challenges related to the ubiquitous, instantaneous, and indeed wavelength-agile nature of SRS. Therefore, as we will discuss in this paper, a careful fiber design, carefully adjusted to the parameters of the operating laser or amplifier is a necessity in order to combine the many attractions of fiber Raman devices with significant BE [3-8].

A large inner-cladding area and numerical aperture (NA) allows for a high BE, as does a small single-mode core. In [5] we have discussed the area ratio limitation between the inner cladding and core for efficient conversion from the pump into the $1^{\text {st }}$ Stokes, in the absence of spectrally dependent loss (e.g., with a step-index core). The gain for the $2^{\text {nd }}$ Stokes and the nonlinear pump depletion, and therefore the area ratio and BE, is restricted by,

$$
G_{2} / \zeta_{0}=\left(A_{c l} / A_{c o}\right)\left(\lambda_{0} / \lambda_{1}\right)^{2}=\left(a_{c l} / a_{c o}\right)^{2}\left(\lambda_{0} / \lambda_{1}\right)^{2}<8
$$

where $G_{2}$ is the $2^{\text {nd }}$ Stokes gain in $\mathrm{dB}, \zeta_{0}$ the nonlinear depletion of the pump induced by the $1^{\text {st }}$ Stokes in $\mathrm{dB}, \lambda_{0}$, and $\lambda_{1}$ are the wavelengths of the Raman pump and $1^{\text {st }}$ Stokes, respectively, and $A_{i}$ is the area and $a_{i}$ the radius of the core and inner cladding (subscript $i=c o$ or $c l$ ). It is possible, but not easy, to relax the condition of (1) by introducing a loss for the $2^{\text {nd }}$ Stokes [6]. The challenge lies in combining a low loss for the $1^{\text {st }}$ Stokes with a useful loss for the $2^{\text {nd }}$ Stokes. The Raman gain grows more quickly than the attenuation of filters at small Raman shifts, so it is not enough to consider the peak of the $2^{\text {nd }}$-Stokes gain. We have found a Raman shift of $80 \mathrm{~cm}^{-1}$ (i.e., much smaller than the $\sim 440 \mathrm{~cm}^{-1}$ shift to the gain peak) to be particularly challenging. Simulations showed that spectral bendloss-filtering in an $18 \mu \mathrm{m}$ diameter W-type core operating at $1660 \mathrm{~nm}$ allowed for an area ratio of 34 [6]. This was an optimized design that maintained sufficiently sharp spectral bendloss characteristics [9], which becomes increasingly difficult for larger cores. The corresponding inner-cladding diameter is $\sim 100 \mu \mathrm{m}\left(\operatorname{area} \sim 8000 \mu \mathrm{m}^{2}\right)$. This is attractive in that it opens up for pumping through a tapered fiber bundle.

There are however several additional factors that limit the BE and the inner-cladding size. Thus, undesired $2^{\text {nd }}-$ order Stokes generation can occur even with area ratios that satisfy (1), if there are pump modes with poor overlap with the desired $1^{\text {st }}$-Stokes core mode [4]. Damage, pulse dispersion, and achievable NA of the inner cladding are other limiting factors [6]. We found that a brightness enhancement of up to 2000 can be possible with realistic fiber parameters, although this depends both on the duration of the pump pulses and their peak intensity $I_{p}$. See Fig. 1. This is much smaller than the five or perhaps even six orders of magnitude of brightness enhancement that a cladding-pumped YDF may allow for [10] (in certain operating regimes), but it is still respectable. Figure 2 presents similar information as Fig. 1 in terms of the inner-cladding size. For pulsed pumping, the fiber length is limited by dispersion of the pump pulse. For a given pump power the inner-cladding area must then be small enough to reach the pump intensity required for efficient Raman conversion in that length. For cw pumping,

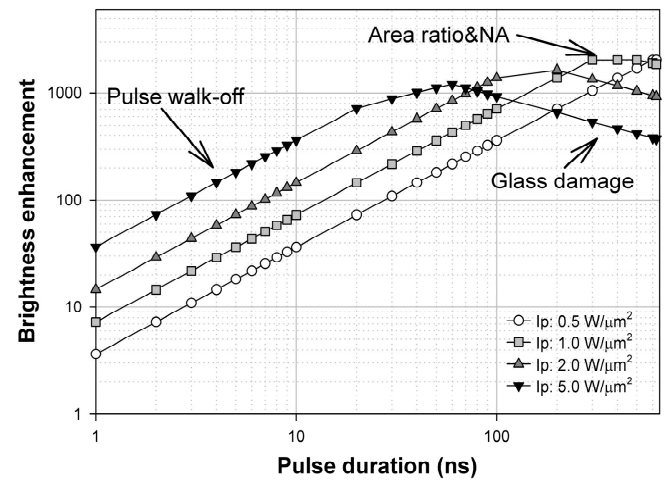

Fig. 1. Limits on brightness enhancement vs. pulse duration at an inner-cladding NA of 0.46 with an optimized W-type 18 m diameter core at different pump intensities. From [6]. 
the background loss rather than dispersion limits the length and thus the inner-cladding size. $2 \mathrm{~dB} / \mathrm{km}$ is assumed in Fig. 2. The graph also incorporates other effects that can limit the inner-cladding size. One line shows the limit set by the $2^{\text {nd }}$ Stokes according to (1), i.e., for an area ratio of eight. Another line shows the maximum inner cladding for an area ratio of 34 , corresponding to the $\mathrm{W}$-type fiber discussed above. The core diameter was $18 \mu \mathrm{m}$ for both the step-index and the $\mathrm{W}$-type fiber. This core diameter will allow for single-mode operation. It may well be possible to operate even larger cores on a single mode, though the long fibers involved may lead to mode-coupling.

To reach the brightness enhancement and the innercladding size of Figs. 1 and 2 it is necessary use a constant pump power, i.e., cw or rectangular pulses. We next consider what happens to the efficiency for mismatched pump powers in the cw and quasi-cw regime, when dispersion can be neglected. Furthermore, we here assume that the background loss of the fiber is sufficiently low to

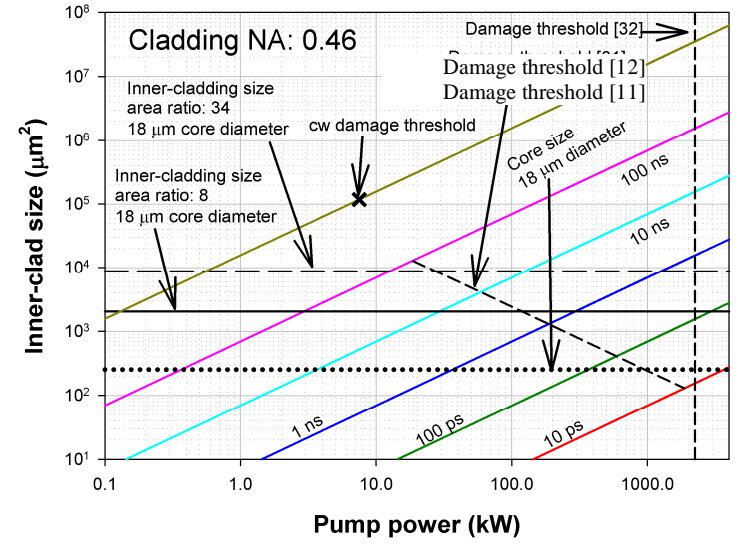

Fig. 2. Limits on the inner-cladding size with 0.46 inner-cladding NA for different pulse durations and pump powers. The dispersion-limited lengths that can be tolerated for efficient $1^{\text {st }}$ Stokes generation become 0.001, 0.096, 0.963, 9.626, $96.258 \mathrm{~m}$ for $10 \mathrm{ps}, 100 \mathrm{ps}, 1 \mathrm{~ns}, 10 \mathrm{~ns}$ and $100 \mathrm{~ns}$ pulses. For the $\mathrm{cw}$ case the tolerable loss is $2 \mathrm{~dB} / \mathrm{km}$.

allow for efficient Raman conversion at the kW-level. Thus, background loss is neglected. Since we can readily make the core large enough to avoid damage at this power-level, we disregard damage, as well. It is then the appearance of the $2^{\text {nd }}$ Stokes that limits the conversion to the $1^{\text {st }}$ Stokes. Fig. 3 shows the $1^{\text {st }}-$ Stokes power $v s$. pump power for different area ratios, without spectral filtering. The fiber length is optimized to reach the highest conversion efficiency for a pump power of $1 \mathrm{~kW}$. For an excessive area ratio of 12 , the $1^{\text {st }}$-Stokes power decreases sharply above the optimal $1 \mathrm{~kW}$ pump power. The same is true for an area ratio of eight, although this does allow for a high conversion efficiency at the optimal power. We conclude that at this area ratio it is imperative to operate with the right pump power in order to reach a good conversion efficiency. For smaller area ratios, and in particular for unity area ratio, the $1^{\text {st }}$-Stokes power continues to grow even beyond the $1 \mathrm{~kW}$ of pump power that yields the highest conversion efficiency. This makes the power-level less critical.

Experimentally, we have demonstrated CP fiber Raman lasers and high-gain amplifiers at around $1 \mu \mathrm{m}$ and $1.6 \mu \mathrm{m}$, cw $([3,4,13])$ as well as pulsed $[1,4,5,8]$ with diffractionlimited output or close thereto. We recently reached $100 \mathrm{~W}$ of cw output power [13] as well as $1 \mathrm{~mJ}$ of energy in the pulsed regime [8]. In addition, an all-fiber source has been reported by others [14] albeit with a low conversion efficiency of $\sim 6.6 \%$.

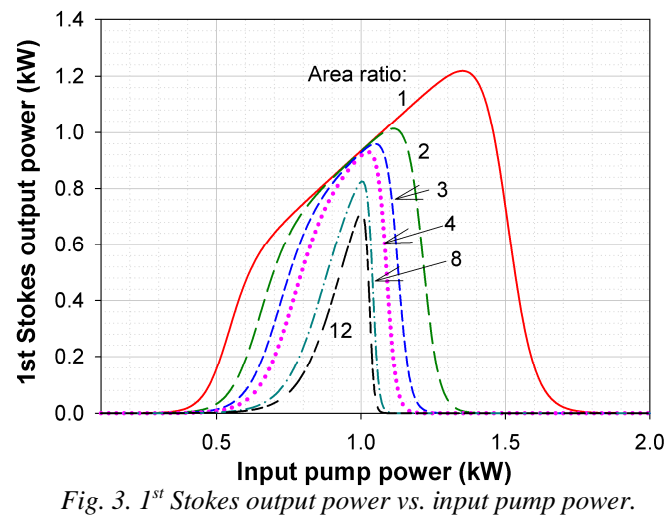

This work was funded by the EPSRC, grant EP/E027318/1.

\section{References}

1. J. Nilsson, J. K. Sahu, J. N. Jang, R. Selvas, D. C. Hanna, and A. B. Grudinin, “Cladding-pumped Raman amplifier”, Proc. Opt. Amplifiers and Their Applications, PD2, Vancouver Canada, July 2002

2. R. R. Rice, "Multimode Raman fiber amplifier and method", US pat. no. 6,353,087 (2002)

3. C. A. Codemard, P. Dupriez, Y. Jeong, J. K. Sahu, M. Ibsen, J. Nilsson, "High-power continuous-wave...”, Opt. Lett. 31, 2290-2292 (2006)

4. C. A. Codemard, High-power cladding-pumped Raman and erbium-ytterbium doped fibre sources, PhD Thesis, U. Southampton (2007)

5. J. Ji, C. A. Codemard, M. Ibsen, J. K. Sahu, and J. Nilsson, “Analysis of the conversion to the first Stokes in cladding-pumped fiber Raman amplifiers", IEEE J. Sel. Top. Quantum Electron. 15, 129-139 (2009)

6. J. Ji, C. A. Codemard, and J. Nilsson, "Brightness enhancement limits in pulsed cladding pumped fiber Raman amplifiers", Fiber lasers VII: technology, systems, and applications, Proc. SPIE 7580, 75801L-75801L-8 (2010)

7. J. E. Heebner, A. K. Sridharan, J. W. Dawson, M. J. Messerly, P. H. Pax, M. Y. Shverdin, R. J. Beach, C. P. J. Barty, "High brightness, quantum-defect-limited conversion efficiency in cladding-pumped Raman fiber amplifiers and oscillators," Opt. Express 18, 14705-14716 (2010) 8. J. Ji, C. A. Codemard, Jayanta K. Sahu, J. Nilsson, "Design, performance, and limitations of fibers...", Opt. Fiber Technol., in press (invited) 9. J. Kim, P. Dupriez, C. Codemard, J. Nilsson, J. K. Sahu, “Suppression of stimulated Raman scattering...”, Opt. Express 14, 5103-5113 (2006) 10. J. Nilsson, "Recent progress and limiting factors in high power fiber laser technology", Proc. Conf. Lasers and Electro-Optics, CTUC1 (2010) 11. B. C. Stuart, M. D. Feit, S. Herman, A. M. Rubenchik, B. W. Shore, M. D. Perry, Phys. Rev. B 53, 1749-1761 (1996)

12. A. V. Smith, B. T. Do, "Bulk and surface laser damage of silica by picosecond and nanosecond pulses...", Appl. Opt. 47, 4812-4832 (2008)

13. C. A. Codemard, J. Ji, J. K. Sahu, and J. Nilsson, 100 W CW cladding-pumped Raman fiber laser at 1120 nm, in Fiber lasers VII: technology, systems, and applications, K. Tankala, Ed., Proc. SPIE 7580, 75801N-75801N-7 (2010)

14. A. K. Sridharan, J. E. Heebner, M. J. Messerly, J. W. Dawson, R. J. Beach, and C. P. J. Barty, Opt. Lett. 34, 2234-2236 (2009) 